\title{
Development of an ATCA IPMI Controller Mezzanine Board to be used in the ATCA developments for the ATLAS Liquid Argon upgrade
}
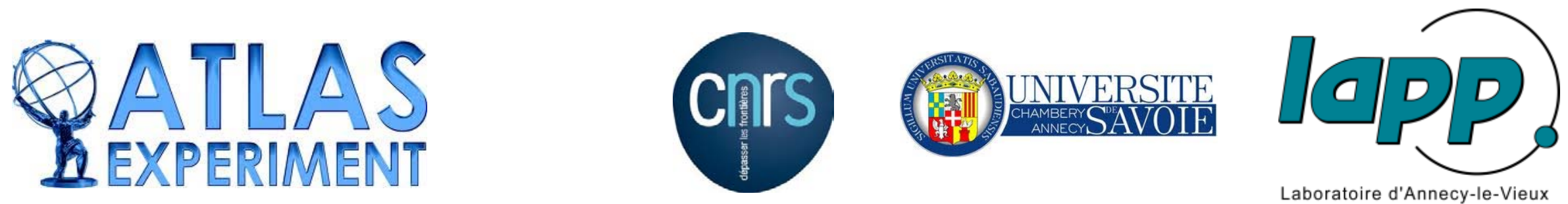


\section{Outline}

- Context

- Motivations

- ROD evaluator

- ATCA test board

- ATCA controller mezzanine

- Summary 


\section{Motivations}

We need :

- High input and output bandwidth

- High speed communications between boards

- Powerful signal processing

This is why ATCA platform was chosen:

- High speed and high density communications between boards over backplane

- Large boards (32×28 cm)

- Possibility of rear transition modules (RTM), and mezzanines card (AMC)

- Reliability (hot swap, real time diagnostic)

We build an ATCA board to evaluate:

- ATCA specifications (Intelligent Platform Management Interface 'IPMI' facilities)

- Management trough Ethernet (Firmware upgrade, DSP configuration, monitoring...)

- Many 12x10Gbps incoming optic fibers

- High speed and high density board.

- High power DSP cells from FPGA

For that we have designed a board called "ROD evaluator" and an IPM Controller Mezzanine board 


\section{ROD evaluator}

\section{Synoptic}

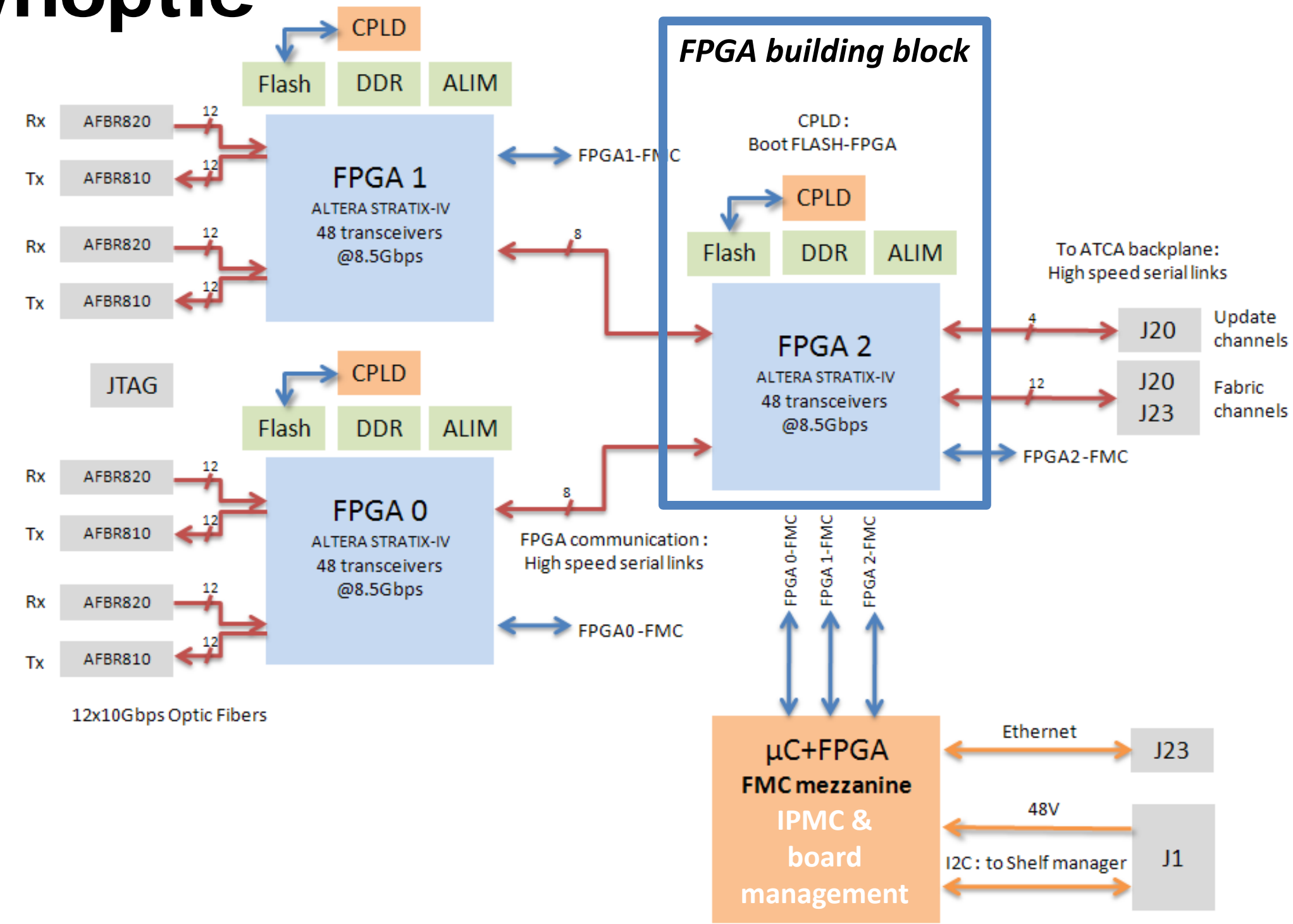




\section{ROD evaluator Design}

\section{CAD completed :}

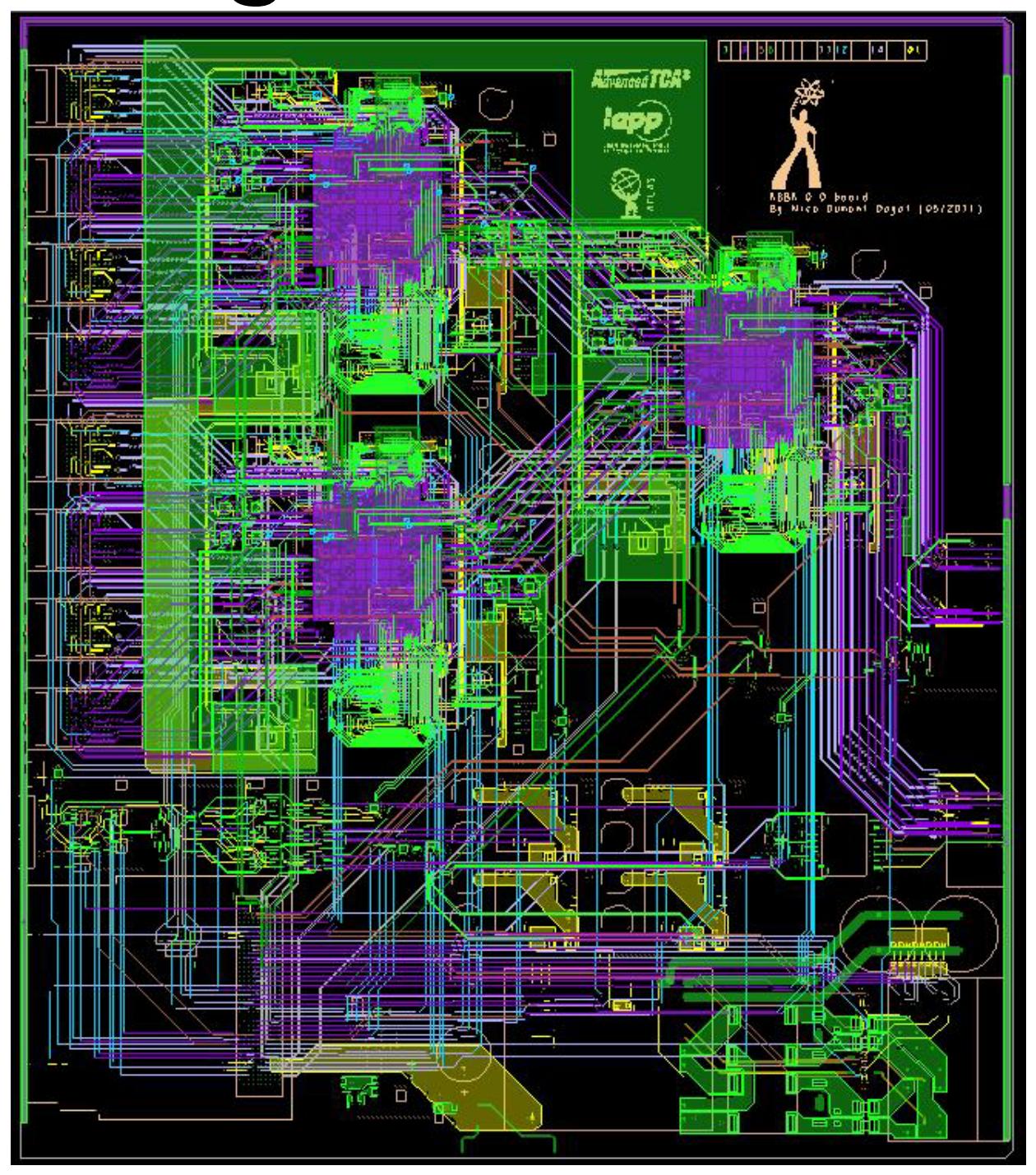

- 16 layers

- Minimum lines width : $75 \mu \mathrm{m}$

- Laser and blind buried vias

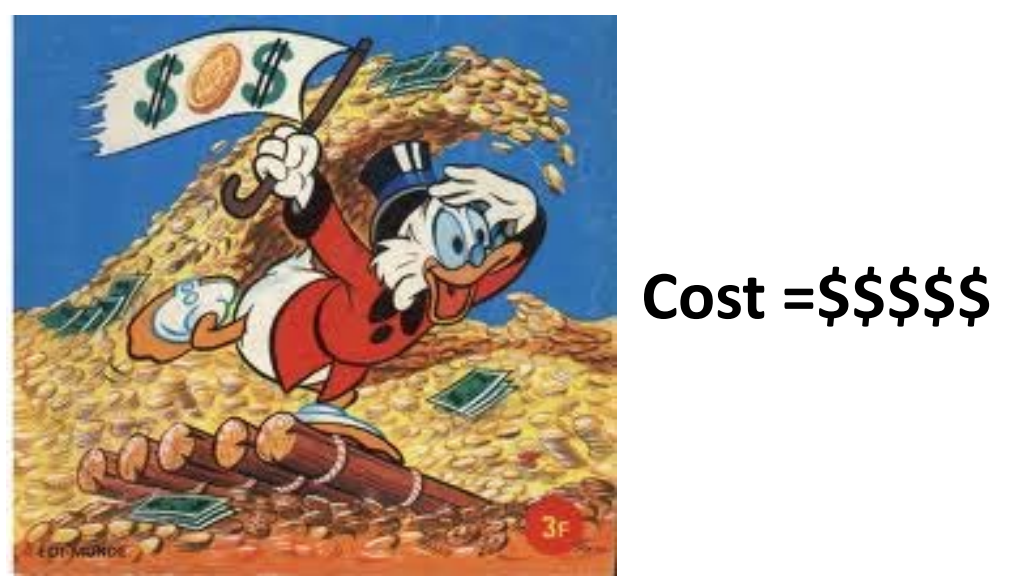

Before launching production :

-> Test FPGA building blocks

$\rightarrow$ Test FMC mezzanine with IPMC We have designed 2 boards: -> ATCA controller mezzanine board -> ATCA test mother board 


\section{ATCA test board Synoptic}

\section{Tests of :}

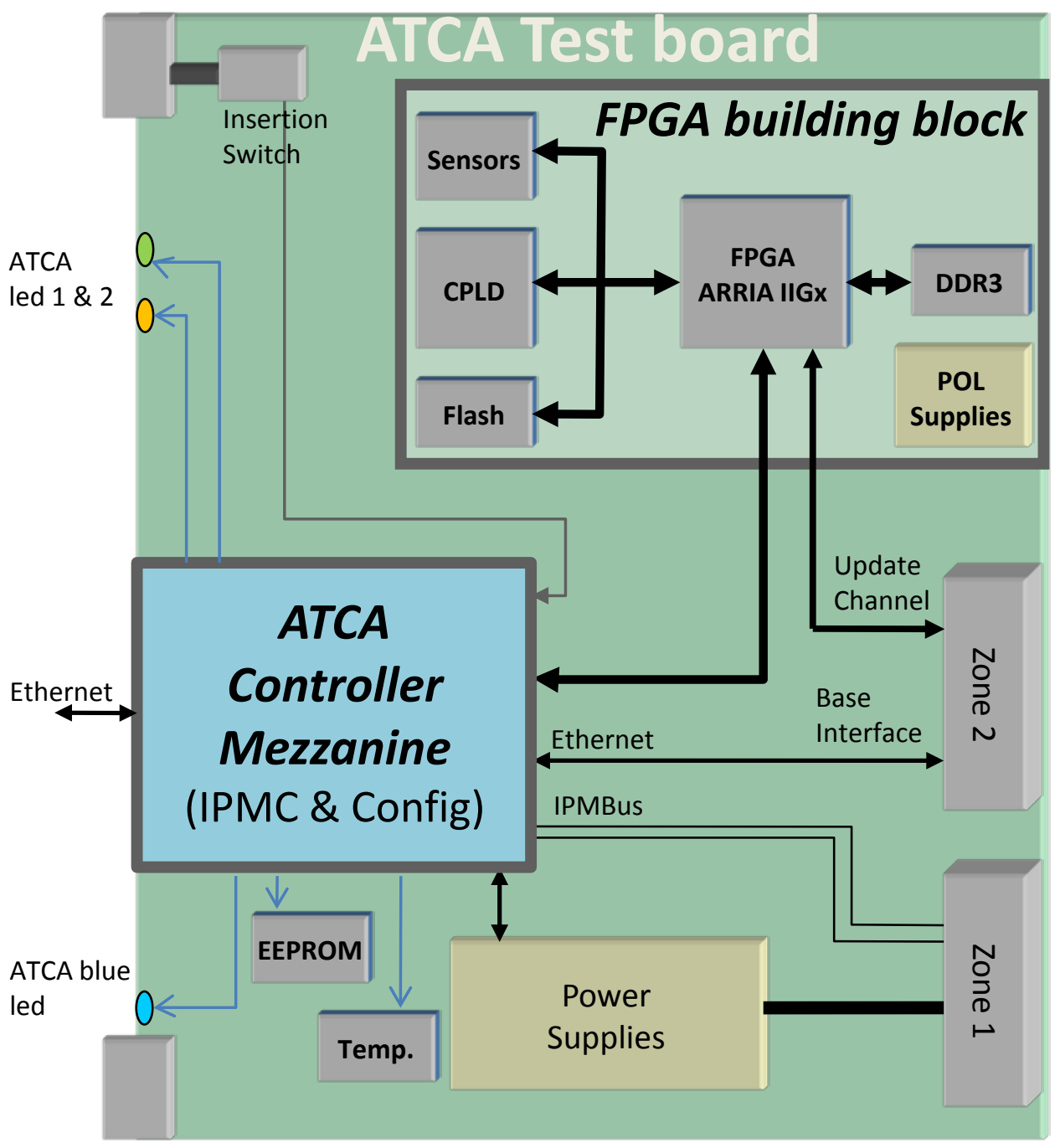

\section{FPGA building block}

- Boot from CPLD \& parallel Flash

- Communications with DDR3 \& Flash

\section{ATCA CTRL Mezzanine :}

- IPMC (IPM Control) trough IPM Bus

$=>$ Communication with Shelf manager

- ATCA power supplies management

$=>$ Hot swap (insertion switch)

$\Rightarrow$ Enable DC/DC

- Alarm/failure diagnostic

- Board configuration through Ethernet

$=>$ Firmware upload

=> Optimal filtering coefficient upload

$=>$ Sensor reading

=> Etc. 


\section{ATCA test board}

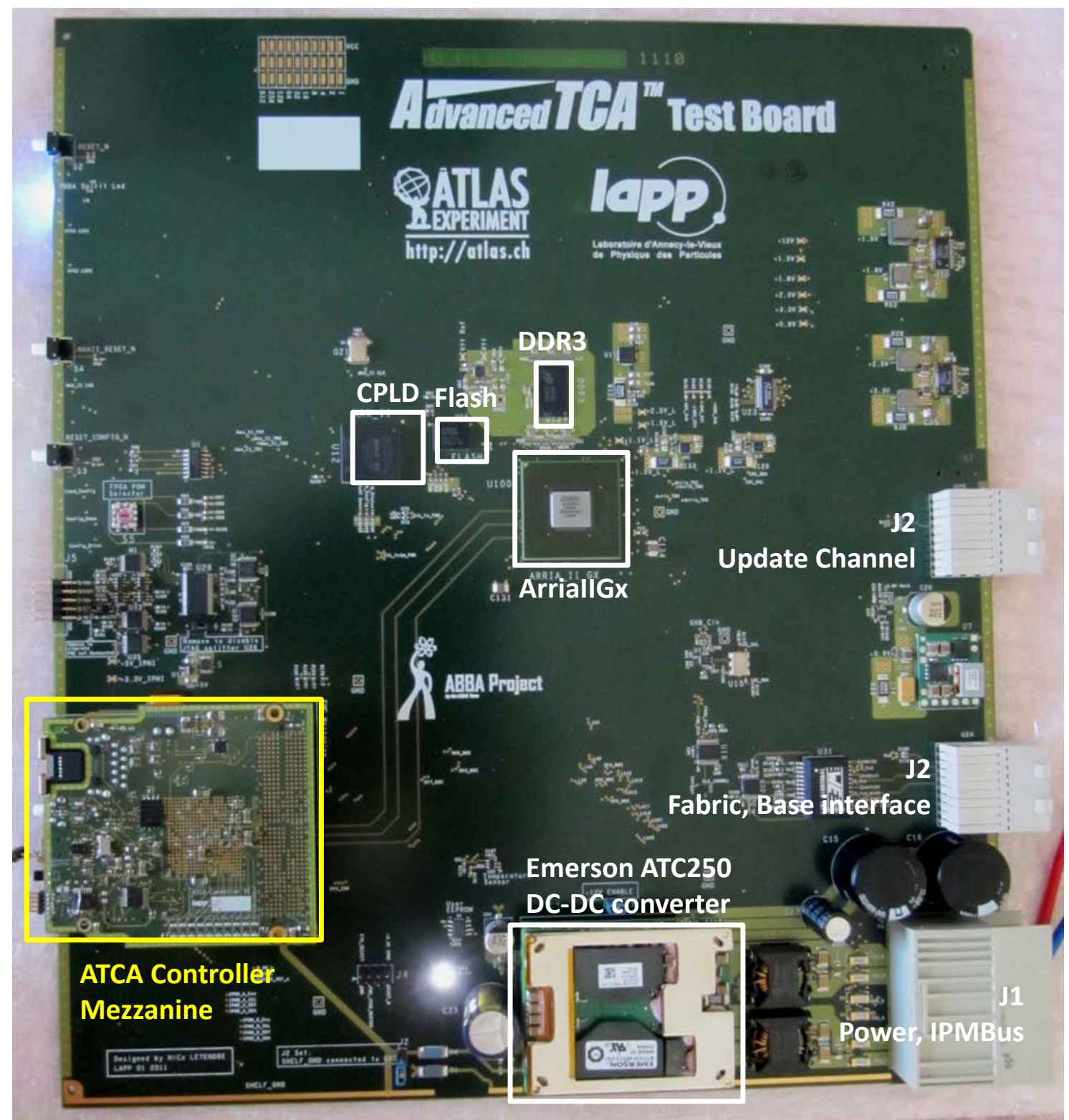




\section{ATCA Controller mezzanine Software}

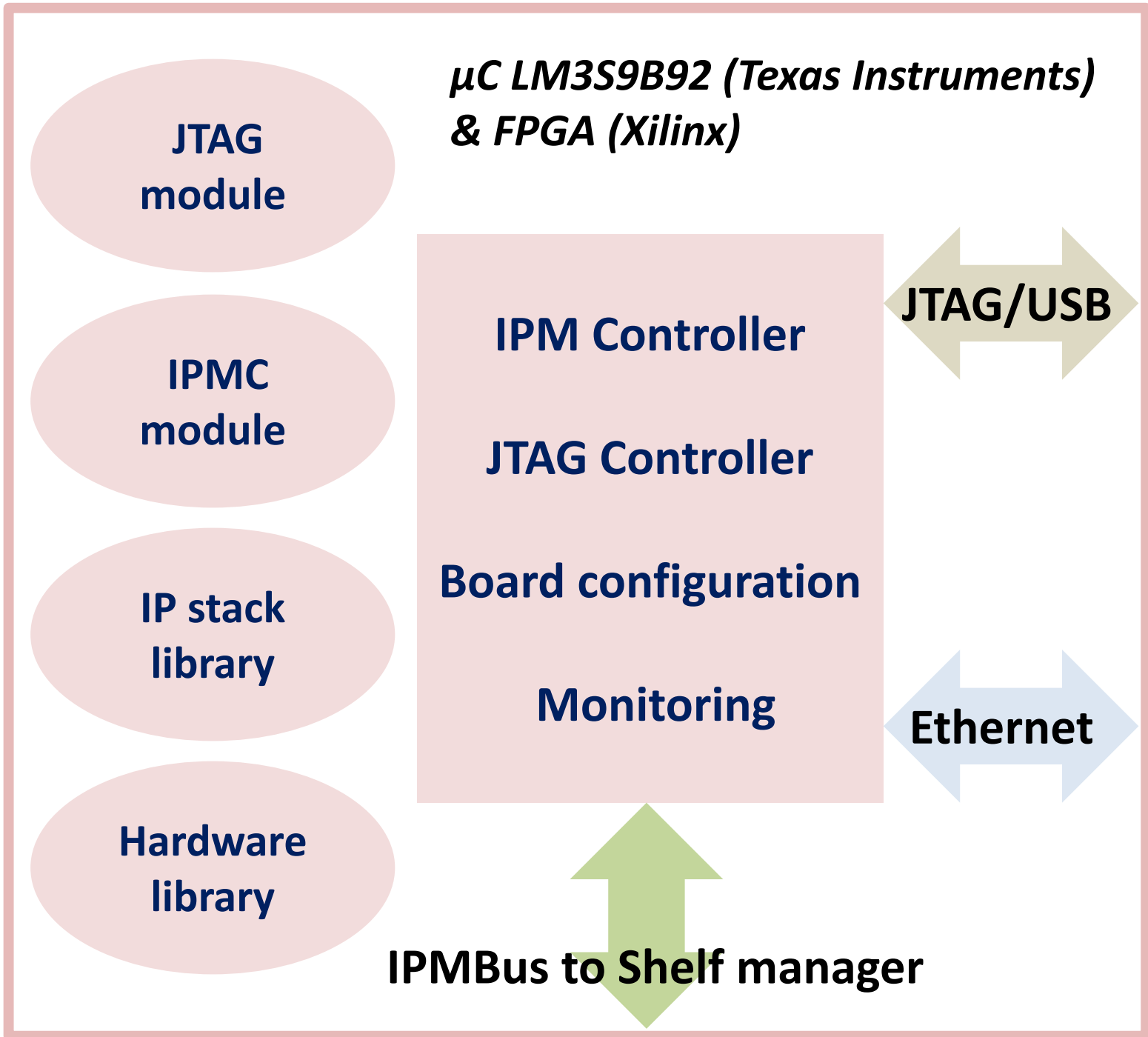

Development tools : LINUX

Compiler Gnu GCC : ARM

Debugger GNU GDB

\& OpenOCD

Programmer T.I

User environment

Web interface

TCP/IP client interface

File server

(boot and board config.) 


\section{ATCA Controller mezzanine FRU \& SDR generator}

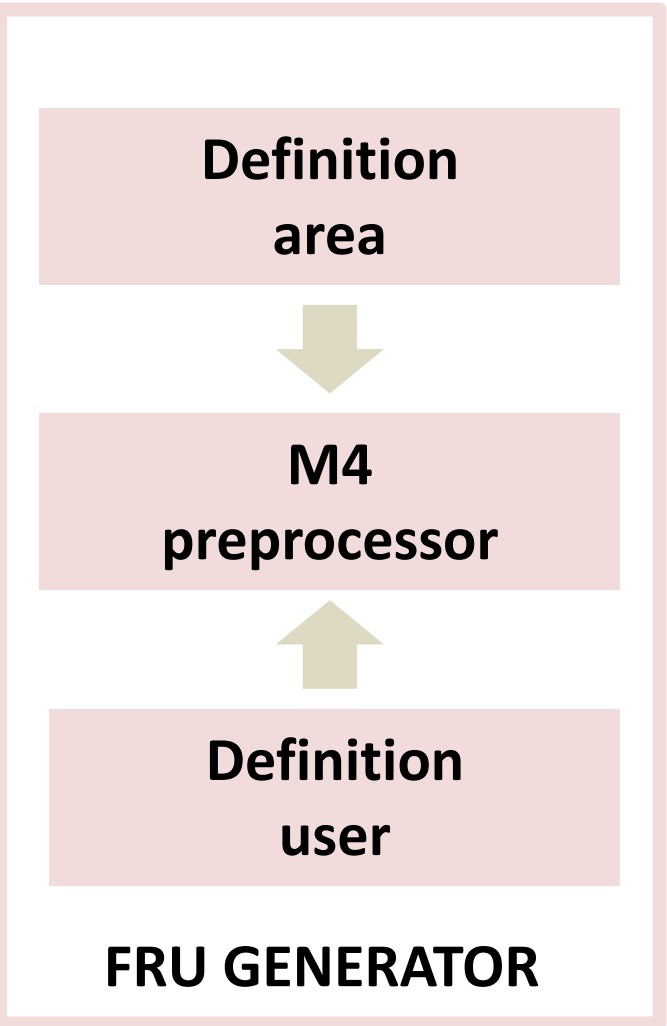

FRU (Field Replaceable Unit) data :

- Identity of the carrier board

=>Ex : IO description on J2 connector SDR (Sensor Data record) :

- Sensors description of the carrier board $=>$ Ex : handle switch, temperature sensor

File to

EEPROM 


\section{ATCA Controller mezzanine Example of info to Shelf manager}

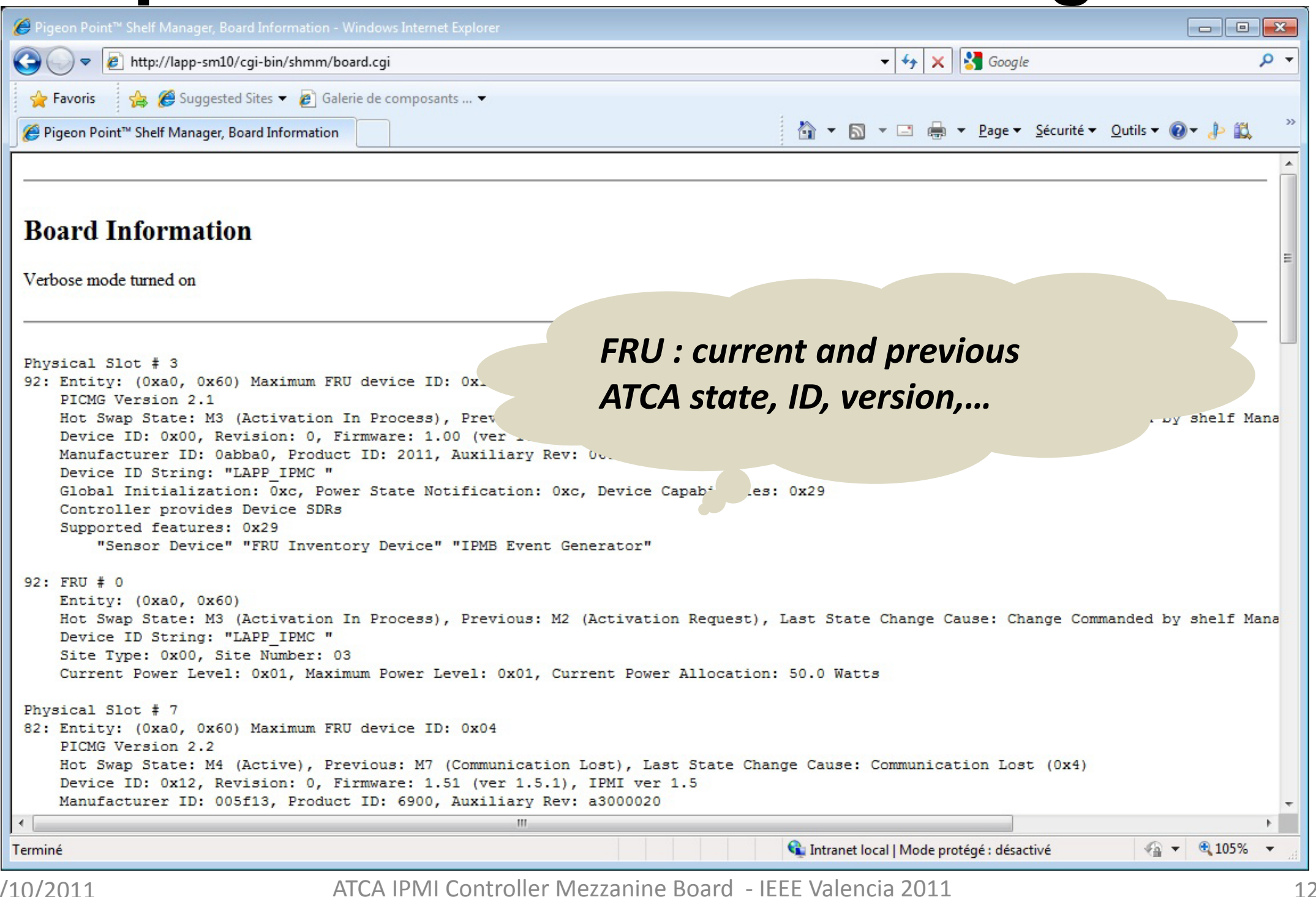




\section{Summary}

We have designed an ATCA ROD Evaluator board to evaluate the functionalities needed by the next generation ROD in the context of the ATLAS LAR upgrade:

- Large number of fast links (High speed and high density board)

- Recent generation of FPGA (many DSP cells and Ser-Des) and optical receivers

- ATCA platform

For the control of that board, we have designed a generic ATCA IPMI Controller mezzanine in the FMC format.

- Acts as an IPM Controller

- Direct Ethernet access

- Uploads the FPGA firmware and other parameters

- Monitors the board parameters

This mezzanine and its software is currently tested on an ATCA Test board we have designed

Next step is the design of a smaller IPM Controller 